\title{
Le syndrome de Cockett
}

\section{Caroline Menez}

CHU Grenoble-Alpes, Clinique universitaire de médecine vasculaire, France <cmenez@chu-grenoble.fr>

Résumé. Cela fait plus de 50 ans que May et Thurner, sur des séries autopsiques, puis Cockett et Thomas, sur des séries phlébographiques, ont décrit ce piège vasculaire veineux du syndrome de Cockett. Celui-ci concerne principalement la veine iliaque primitive gauche, piégée entre l'artère iliaque primitive droite et le mur antérieur vertébral. Cette compression serait, en soi, un facteur de thrombose et expliquerait la localisation gauche prédominante des événements thrombotiques. Les années 1990 ont vu se développer les techniques endovasculaires veineuses rendant possible le traitement de ce syndrome. Les séries publiées sont maintenant nombreuses. Cependant, les données sont extrêmement hétérogènes et aucune stratégie consensuelle n'est à ce jour proposée. Les circonstances qui conduisent au diagnostic sont variées : thrombose aiguë, syndrome post-thrombotique, voire patients symptomatiques hors de tout contexte thrombotique. Sur le plan diagnostique, les méthodes d'évaluation sont nombreuses mais aucune n'est spécifiquement validée. Les résultats des séries rétrospectives sont très favorables à la prise en charge interventionnelle des obstructions veineuses thrombotiques aiguës ou chroniques, mais des critères objectifs, notamment pour poser l'indication du déploiement d'une endoprothèse, restent à définir. En l'absence de thrombose, établir un lien de causalité entre une symptomatologie clinique et des données d'imagerie en faveur d'un syndrome de Cockett peut s'avérer difficile. La détermination de critères permettant d'établir ce lien est un prérequis indispensable à toute proposition de traitement interventionnel dans ce contexte.

Mots clés : compression, sténose, thrombose

\section{Abstract \\ May-Thurner syndrome}

More than 50 years ago, May and Thurner, on autopsy series, then Cockett and Thomas, on phlebographic series, described this venous vascular trap of the May-Thurner syndrome. It concerns most of the time the left primary iliac vein, trapped between the right primitive iliac artery and the anterior vertebral wall. This compression would be a factor of thrombosis and would explain the predominant left localization of the thrombotic events. The 1990s saw the development of venous endovascular techniques and thus the possibilities of treatment of this syndrome. There are many publications regarding this treatment. However, data are extremely heterogeneous, and no consensual strategy has yet been proposed. The circumstances leading to the diagnosis are varied: acute thrombosis, post-thrombotic syndrome, even symptomatic patients out of any thrombotic context. They are many methods to explore deep venous obstruction but none is specifically validated. Despite this heterogeneity, published series show very good results in terms of technical success, rate of permeability or clinical improvement. Endovascular treatment has been shown to be effective and safe in retrospective case series, but objective criteria for the indication of the deployment of an endoprosthesis remain to be defined. In the absence of thrombosis, establishing a causal link between clinical symptomatology and imaging data in favor of May-Thurner syndrome may be difficult. Determining criteria to establish this link is an essential prerequisite before proposing invasive therapy.

Tirés à part : C. Menez
Key words: compression, stenosis, thrombosis 
Le syndrome de Cockett (SC) appartient à la famille des pièges veineux. Il s'agit le plus souvent de la compression de la veine iliaque primitive gauche au sein d'une pince artériovertébrale, formée en avant par l'artère iliaque primitive droite, et en arrière par le mur vertébral antérieur et, plus précisément, celui de la cinquième vertèbre lombaire. Des variations anatomiques existent, avec différents niveaux de compression de l'axe veineux, voire des formes droites. Les descriptions princeps remontent aux années 1950 et 1960. May et Thurner travaillaient sur des données autopsiques, en 1956 [1] aux États-Unis, alors que Cockett et Thomas [2] se sont penchés sur des données phlébographiques, en Europe dix années plus tard.

La veine y est décrite comme subissant un phénomène de compression extrinsèque au sein de la pince artériovertébrale, auquel s'ajoutent des microtraumatismes répétés induits par la pulsatilité artérielle. En résulte la prolifération de l'endothélium vasculaire, à l'origine de synéchies ou spurs réduisant progressivement le calibre de la lumière vasculaire.

La stase veineuse, l'hypercoagulabilité et les lésions veineuses sont les trois facteurs de la triade de Virchow prédisposant au processus thrombotique. Virchow, médecin pathologiste, avait par ailleurs décrit la prédominance gauche des thromboses [3].

\section{Présentations cliniques}

Le SC, obstacle mécanique à l'écoulement veineux, favorise la survenue d'une thrombose veineuse profonde aiguë à l'occasion, le plus souvent, d'un facteur provoquant surajouté, à savoir la chirurgie, l'immobilisation, l'introduction d'une pilule ostroprogestative, la grossesse ou le post-partum. La localisation thrombotique au sein de la veine iliaque primitive entraîne un syndrome obstructif proximal à l'origine d'un œdème tendu douloureux du membre inférieur dans son ensemble, dès la racine de cuisse (figure 1). Ainsi, c'est dans ce contexte de thrombose veineuse aiguë proximale, plus ou moins étendue mais incluant l'étage iliaque, que le diagnostic de SC est le plus souvent évoqué.

Il est ensuite suspecté en contexte chronique, devant la persistance d'une symptomatologie veineuse à distance d'un épisode thrombotique. Les symptômes évocateurs sont alors les douleurs et les lourdeurs du membre inférieur gauche, le plus souvent en position debout prolongée, améliorée en position couchée, jambe surélevée. Une claudication veineuse est parfois décrite, avec survenue à la marche d'une sensation de jambe lourde, voire de jambe de bois. L'examen clinique recherche les signes non

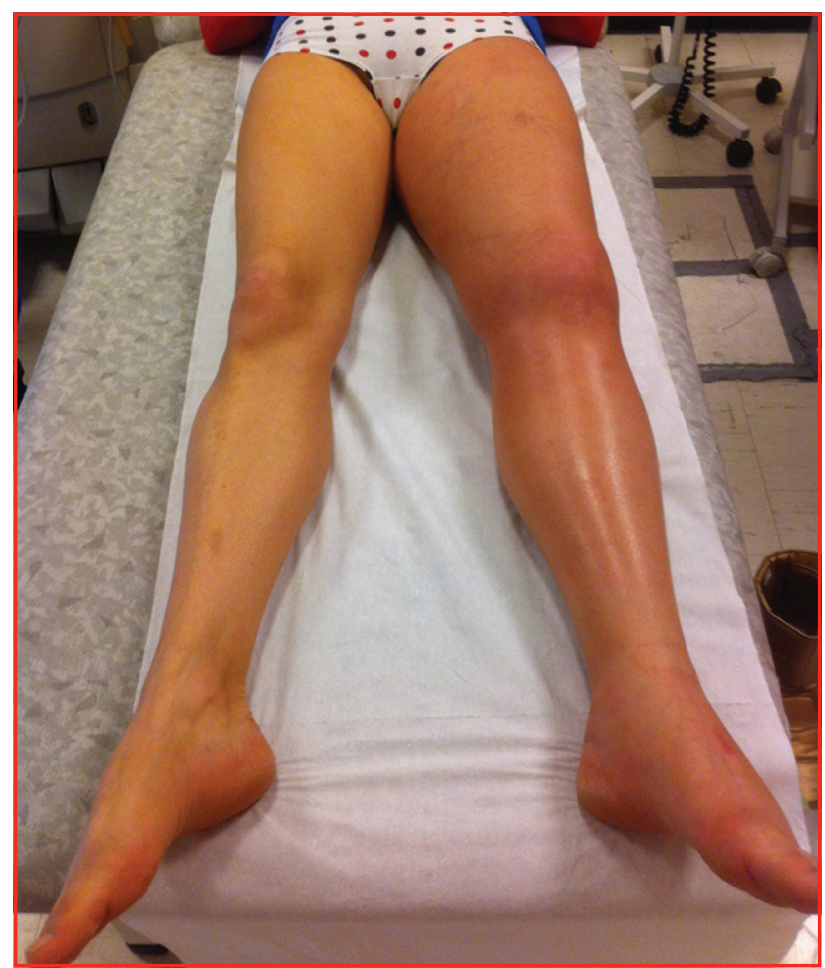

Figure 1. Thrombose veineuse profonde aiguë proximale du membre inférieur gauche.

spécifiques d'insuffisance veineuse chronique : le développement de varices, un œdème (figure 2), des modifications cutanées allant de l'hyperpigmentation à l'ulcère ouvert. L'abdomen et le pelvis doivent être inspectés à la recherche d'une collatéralité abdominale ou d'un réseau sus-pubien. À l'interrogatoire, les antécédents thrombotiques doivent être recherchés. Le score de Villalta, le questionnaire de qualité de vie CIVIQ (ChronIc Venous Insufficiency quality of life Questionnaire) et la classification CEAP (Clinique, étiologie, anatomie, pathophysiologie) peuvent aider le praticien à évaluer les patients dans ce contexte.

Enfin, le SC peut être suspecté devant un tableau clinique similaire à celui précédemment décrit, mais sans aucun antécédent thrombotique personnel. Affirmer le diagnostic de SC et établir le lien de causalité entre ce syndrome et la symptomatologie clinique peuvent alors s'avérer complexes.

Ainsi, différentes situations cliniques peuvent conduire le praticien à suspecter la présence d'un SC, qui est par ailleurs le plus souvent asymptomatique. Sa prévalence reste donc mal définie et est probablement sous-estimée.

\section{Données d'imagerie}

Contrairement au système artériel, il n'existe pas de critère validé permettant de quantifier une sténose veineuse. 


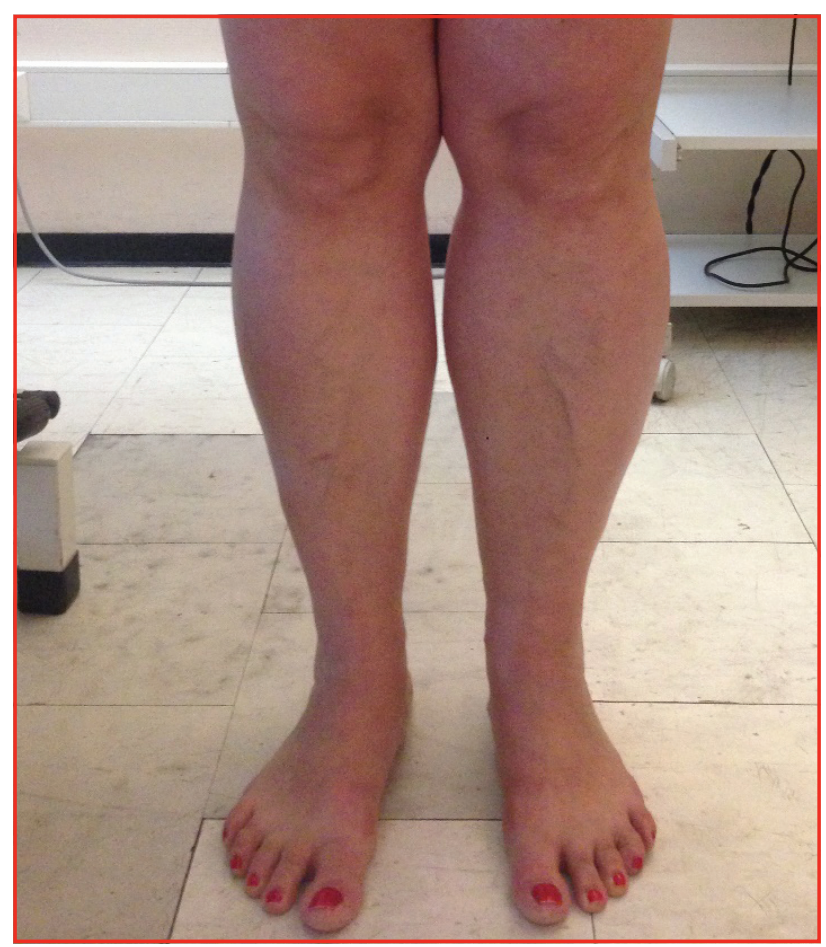

Figure 2. Syndrome post-thrombotique du membre inférieur gauche. CEdème du mollet gauche.

La compliance veineuse complique l'interprétation de la seule mesure antéropostérieure du vaisseau en coupe. En effet, une veine peut apparaître comprimée dans une dimension, mais conserver cependant une surface circulante tout à fait normale. Ainsi, l'analyse morphologique du vaisseau peut conduire à des diagnostics faussement positifs et différents auteurs s'accordent à dire qu'il est plus fiable d'étudier l'hémodynamique de l'axe veineux.

Une fois la sténose retenue sur les données d'imagerie, la seconde étape est d'établir une corrélation entre cette sténose, son expression clinique et son risque thrombotique induit.

Des équipes ont comparé des données scanographiques et cliniques, s'intéressant pour l'une à la corrélation entre sténose veineuse iliaque et antécédent thrombotique homolatéral, et pour l'autre au taux de sténose confirmé au sein de populations symptomatiques versus asymptomatiques $[4,5]$. Il s'agit certes de petites séries, mais ces travaux montrent bien la difficulté à établir un lien clair entre clinique, antécédents thrombotiques et données d'imagerie. Ainsi, une compression décrite et retenue en imagerie peut être totalement asymptomatique, ou parfois découverte en bilan d'une insuffisance veineuse chronique voire d'un événement thrombotique aigu.

L'échographie doppler (ED) est l'examen de première intention d'exploration du système veineux et donc de la recherche d'une compression veineuse. L'ED permet une analyse morphologique, mais également hémodynamique par l'étude des vitesses de flux et de leur direction. L'étude de l'étage iliocave se fait à l'aide d'une sonde basse fréquence, en mode $2 \mathrm{~B}$, couleur ou énergie. Des manœuvres dynamiques peuvent permettre de sensibiliser l'examen. Une fois positionnée, la sonde en coupe transversale sur la veine cave inférieure sous-rénale, un mouvement de translation de celle-ci permet de dégager en avant les artères iliaques, et en arrière le corps vertébral. La veine iliaque primitive gauche se déroule en regard du corps vertébral cheminant au sein de l'espace artériovertébral. Si cette veine est facilement dégagée, de gros calibre, sans effet d'aliasing ou d'accélération hémodynamique segmentaire, le diagnostic de SC peut a priori être exclu (figure 3). La position couchée couplée à l'appui de la sonde sur l'abdomen peut contribuer à un diagnostic faussement positif. La seule mesure du calibre antéropostérieur de la veine iliaque primitive gauche au sein de la pince artériovertébrale en position couchée est donc insuffisante. Une mesure du calibre maximal obtenu lors d'une manœuvre de Vasalva est intéressante mais de réalisation difficile. Il est possible d'obtenir un résultat comparable en demandant au patient la réalisation d'une expiration contre résistance ou par un mouvement de compression puis relâchement de la veine iliaque primitive gauche à l'aide de la sonde et la mesure du calibre maximal obtenu à l'issu de ce mouvement. Il n'existe pas un critère diagnostique mais des arguments en faveur, parmi lesquels : des critères directs de sténose tels que la réduction de calibre veineux, l'impossibilité de visualiser la veine, un effet d'aliasing segmentaire, une accélération hémodynamique des vitesses. Les critères indirects d'une compression significative sont : une dilatation des axes veineux en amont avec un ralentissement significatif des flux, une perte de modulation respiratoire, une inversion des flux notamment l'iliaque interne (figure 4), ainsi que la présence d'une collatéralité. Lorsque l'ED est réalisée en contexte aigu de thrombose veineuse profonde, le diagnostic peut se révéler impossible en raison de la dilatation de la veine par le thrombus lui-même (figure 5). Le SC est mis en évidence après aspiration du thrombus en cas de prise en charge interventionnelle. En cas de séquelles chroniques post-thrombotiques, la veine apparaît partiellement ou totalement occluse, souvent rétractile.

L'exhaustivité est parfois limitée à l'étage abdominal en cas de surpoids. Dans ce cas, la réalisation de l'examen à jeun est souhaitable. La principale difficulté du diagnostic du SC reste cependant l'absence de critère sémiologique de référence.

Le phléboscanner (figure 6) est réalisé par injection bipédieuse de produit de contraste dilué ( $150 \mathrm{mg} / \mathrm{mL}$ d'iode) 


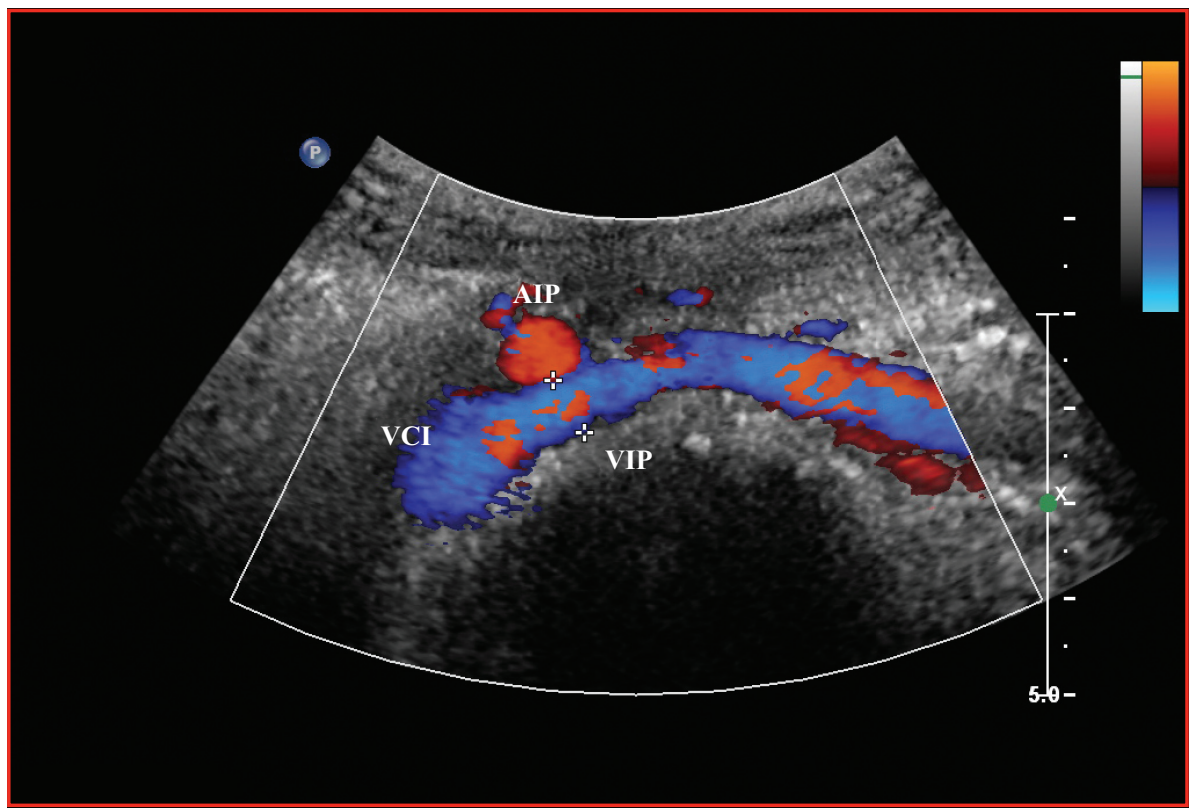

Figure 3. Échographie en mode énergie : veine iliaque primitive gauche (VIP) de bon calibre en arrière de l'artère iliaque primitive droite (AIP). Veine cave inférieure (VCl) sous-rénale perméable.

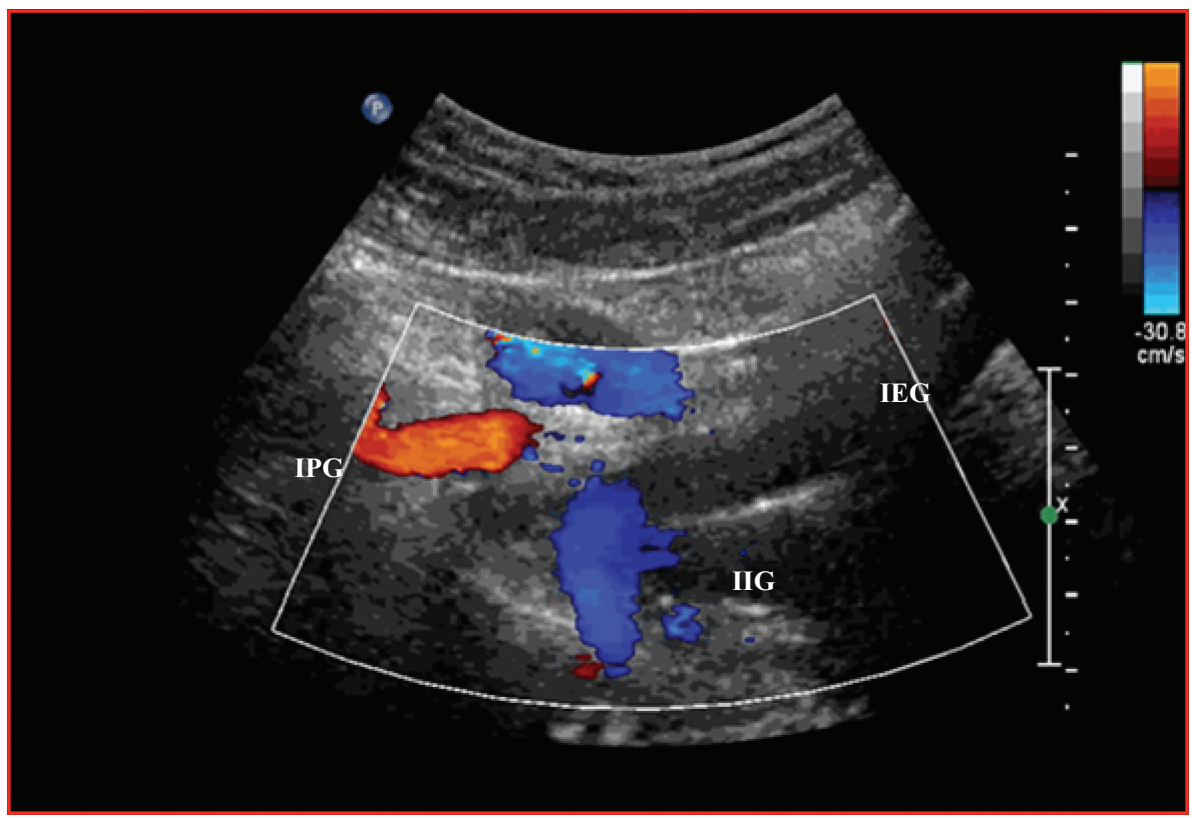

Figure 4. En échographie doppler, inversion de flux au sein de la veine iliaque interne en faveur d'un obstacle en aval.

et levée progressive de garrots positionnés en racine de cuisse et au niveau sous-poplité. La ponction peut s'avérer difficile notamment en cas de syndrome post-thrombotique évolué. Des bains de pied à l'eau chaude sont parfois utilisés en complément des garrots, voire la ponction sous contrôle échographique. Il permet d'analyser le chenal circulant et les voies de dérivation en cas d'occlusion.
Le phlébo-IRM, par injection de $10 \mathrm{ml}$ de gadolinium dilué à $80 \%$, est techniquement possible mais offre une analyse moins précise.

Un angioscanner conventionnel peut être réalisé en contexte de thrombose veineuse profonde aiguë afin de préciser le pôle supérieur du thrombus, si l'exhaustivité échographique est insuffisante à l'étage iliocave ou en cas de suspicion 


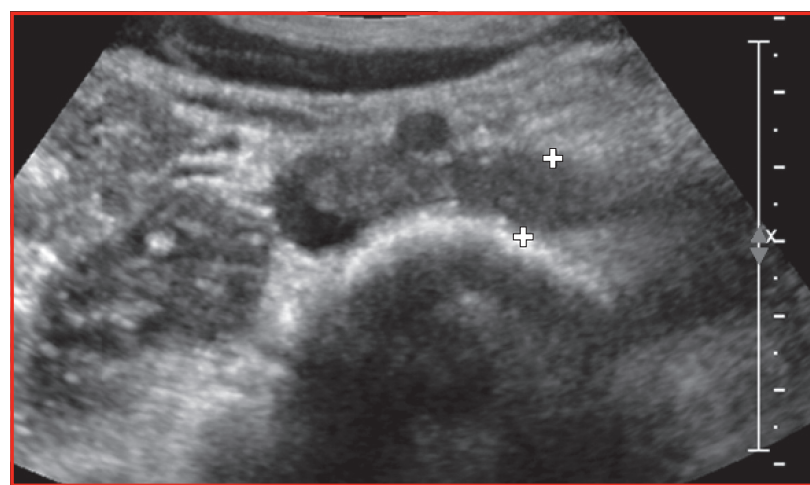

Figure 5. Échographie : thrombose veineuse aiguë iliaque primitive gauche avec débord du thrombus au sein de la veine cave inférieure. Le syndrome de Cockett ne sera dévoilé qu'après aspiration du thrombus.

d'embolie pulmonaire associée. Il n'est, en revanche, pas adapté à l'évaluation des séquelles veineuses au stade de thrombose chronique.

Les données les plus fiables sont celles obtenues lors de la procédure interventionnelle par contrôle veinographique (figure 7). Là encore, la seule mesure des calibres veineux est insuffisante. Les critères indirects sont essentiels, notamment un flux veineux lent en amont de l'obstacle et la présence de voies de dérivation (réseau sus-pubien, lombaire ascendant, pré-sacré ou sous-cutané abdominal) [6]. Les mesures des gradients de pression veineuse sont parfois réalisées pour déterminer le caractère significatif d'une sténose veineuse.

L'IVUS (intra vascular ultra sound) s'est progressivement développé ces dernières années, considéré comme le gold standard par certaines équipes pour l'évaluation des lésions post-thrombotiques et du degré de sténose. C'est une technique onéreuse globalement peu disponible en France en raison du surcoût induit de la procédure. L'IVUS offre une analyse purement morphologique, sans information hémodynamique. Pour certaines équipes, l'IVUS est supérieur à la phlébographie pour l'évaluation du degré morphologique de sténose [7,8] et, notamment, en cas de lésion non thrombotique [9]. Des études, certes un peu anciennes, ont comparé les performances de l'IVUS à la veinographie pour l'évaluation du degré de sténose veineuse, avec une supériorité de l'IVUS mais également des lésions non diagnostiquées par cette technique ou sous-évaluées [10]. La pléthysmographie à air [11] et les mesures de pression veineuse ambulatoire [12] pourraient apporter des arguments en faveur d'une obstruction veineuse, mais ne sont plus réalisées de manière courante.

\section{Prise en charge thérapeutique}

Les années 1990 ont vu le développement des techniques interventionnelles vasculaires veineuses et donc de la possibilité de traitement du SC, quelque peu oublié depuis les

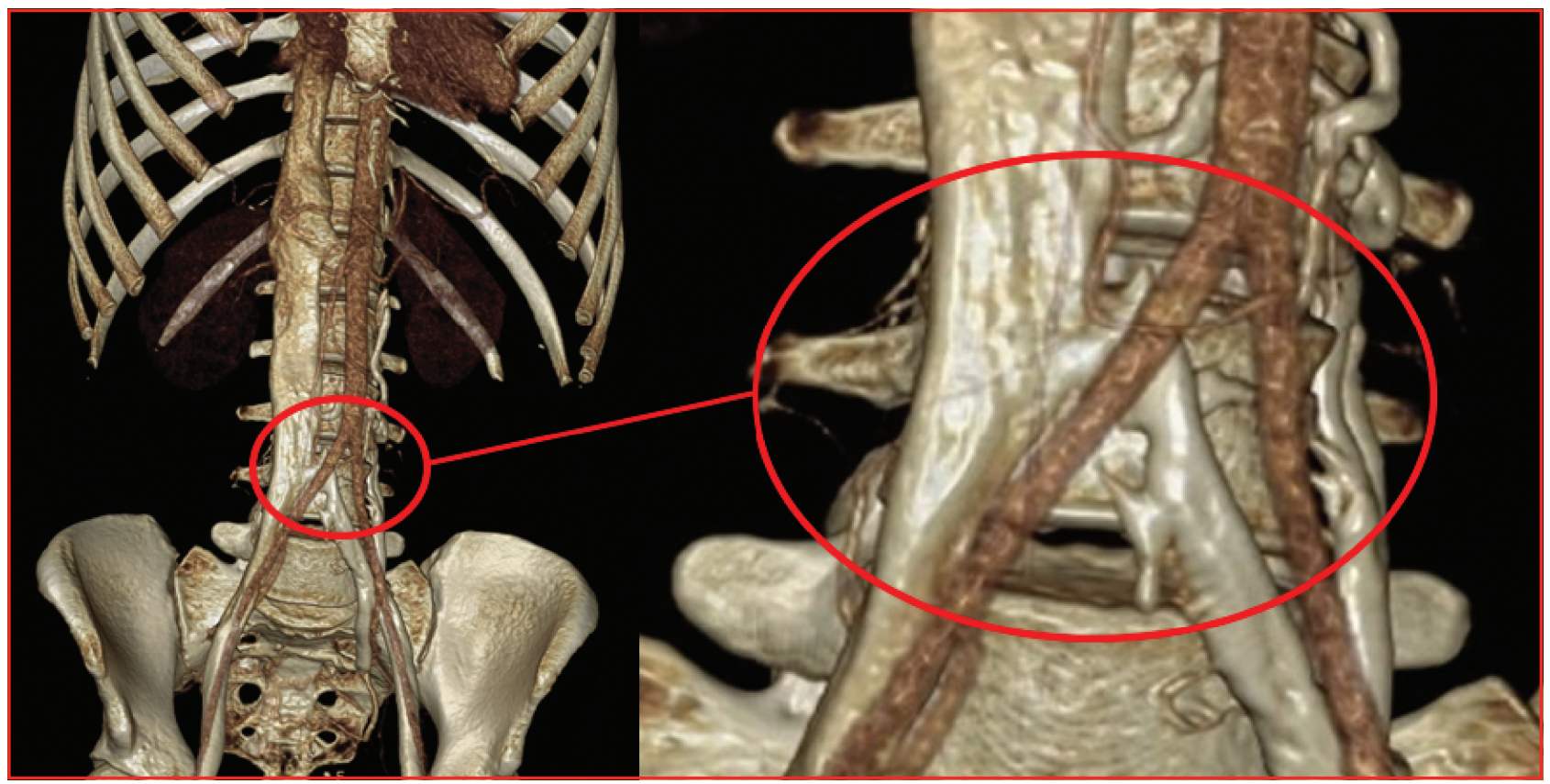

Figure 6. Phléboscanner reconstruit en rendu volumique : compression de l'axe veineux iliaque primitif gauche par l'artère iliaque primitive droite. 


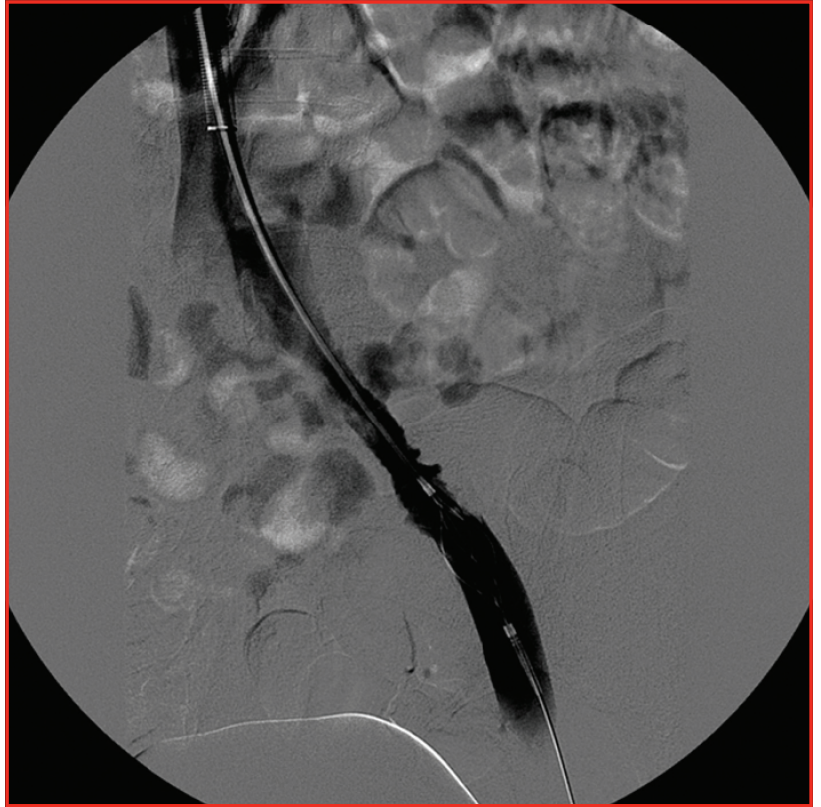

Figure 7. Traitement endovasculaire d'une thrombose veineuse aiguë iliaque primitive gauche par technique pharmacomécanique : persistance d'irrégularités au contrôle veinographique après aspiration de la totalité du thrombus.

descriptions princeps $[13,14]$. La reperméation de l'axe veineux iliaque primitif gauche, objectif du traitement interventionnel du SC, est obtenue par différentes techniques en fonction du contexte dans lequel l'intervention est réalisée. En cas de thrombose veineuse profonde du membre inférieur gauche avec atteinte de l'étage iliaque, un SC peut être suspecté. La pierre angulaire du traitement de la thrombose veineuse reste bien sûr l'anticoagulation. Cependant, un certain nombre de patients sélectionnés ayant accès à un plateau technique et à une équipe experte peuvent bénéficier d'une prise en charge interventionnelle. En attendant le résultat d'études prospectives randomisées, de nombreuses séries suggèrent que ce traitement est sûr et efficace, avec un taux de désobstruction de plus de $50 \%$ chez 83 à $100 \%$ des patients [15]. L'effet sur la réduction du syndrome postthrombotique fait l'objet de l'étude ATTRACT [16].

La fibrinolyse dirigée par cathéter est maintenant remplacée par les techniques pharmaco-mécaniques associant fibrinolyse in situ, aspiration du thrombus et déploiement d'un stent permettant de lever le SC. Ce traitement est réalisé au cours d'une seule et même procédure. La désobstruction doit être optimale, la persistance de thrombus résiduel augmentant la morbidité du syndrome post-thrombotique [17]. L'abord jugulaire doit donc être privilégié, permettant l'accès au réseau veineux dans son ensemble afin d'extraire un maximum de thrombus.

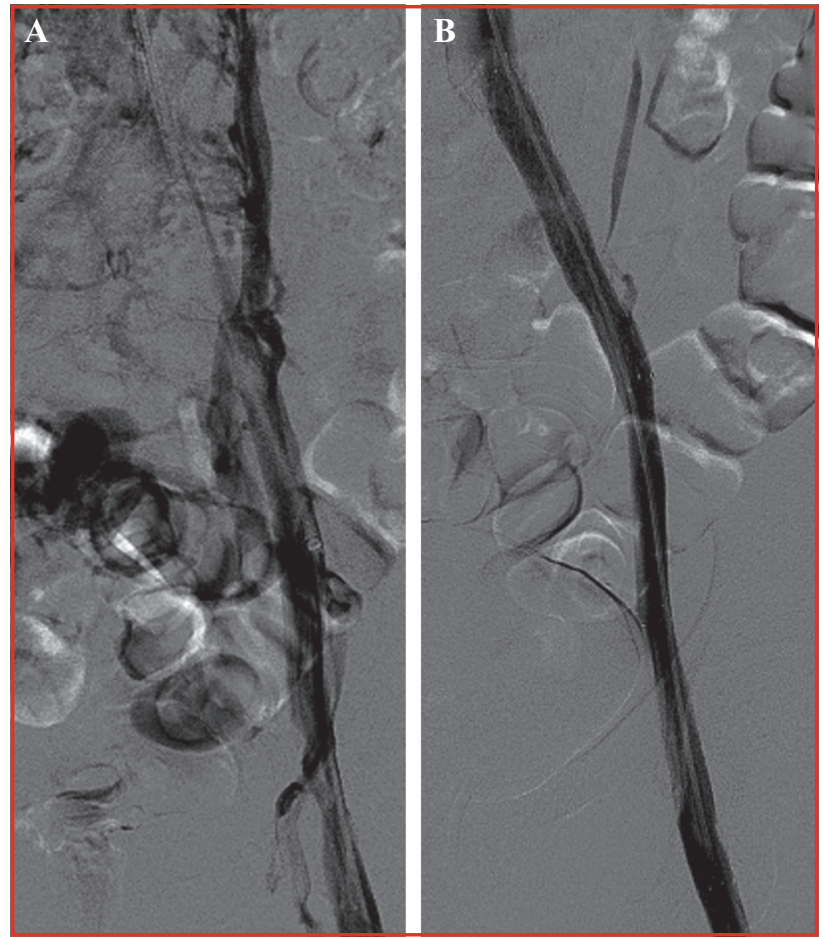

Figure 8. Contrôle veinographique en cours de procédure interventionnelle. (A) Occlusion chronique iliaque primitive gauche et collatérale de drainage. (B) Contrôle après angioplastie et déploiement d'un stent iliaque primitif. La collatérale n'est plus opacifiée.

$\mathrm{Au}$ stade de lésions veineuses chroniques postthrombotiques, il n'est pas rare que les séquelles post-thrombotiques soient étendues et ne concernent pas uniquement le segment veineux iliaque primitif au sein de la pince artériovertébrale. L'angioplastie par dilatation progressive au ballonnet permet de lever les synéchies et de réouvrir progressivement les axes vasculaires, dont le maintien de la perméabilité nécessite le déploiement de stents (figure 8).

De nombreuses séries rapportent les résultats de la prise en charge par angioplastie-stenting de lésions chroniques post-thrombotiques. Les taux de succès technique et de perméabilité rapportés sont élevés, la morbidité faible, et les résultats en termes d'amélioration clinique et de guérison des ulcères satisfaisants [18].

La troisième situation concerne les patients présentant une symptomatologie veineuse chronique au membre inférieur gauche sans antécédent thrombotique, avec une imagerie évocatrice d'un SC. Se posent alors les questions de la responsabilité de la sténose veineuse retenue sur les données d'imagerie et de l'indication d'un traitement interventionnel afin de lever cette sténose.

Les équipes qui interviennent dans ce contexte obtiennent de bons résultats concernant l'amélioration clinique et le 
taux de perméabilité $[14,19,20]$. Ils concluent que ce traitement est à réserver aux stades sévères (CEAP 4 à 6), ou en cas d'échec d'un traitement conservateur pour les stades peu évolués.

Certaines équipes ont, par ailleurs, comparé les résultats d'une prise en charge interventionnelle dans les trois situations précédemment décrites, à savoir patients présentant un SC non thrombotique (patient présentant une symptomatologie clinique et des arguments d'imagerie en faveur d'un SC mais sans survenue d'événement thrombotique), en contexte de thrombose à la phase aiguë et en contexte thrombotique au stade de lésions chroniques. Les meilleurs résultats sont obtenus pour une prise en charge au stade non thrombotique. En cas d'épisode thrombotique, la perméabilité est supérieure au stade aigu, plutôt qu'au stade de séquelles chroniques. Les auteurs concluent au bénéfice d'une prise en charge précoce avant la survenue d'un épisode thrombotique [21]. La réalisation d'un traitement invasif à ce stade reste toutefois discutable au vu du faible niveau de preuve.

Une autre question est celle de la décision de déployer un stent sur une sténose suspectée. Les difficultés concernant les critères diagnostiques ont déjà été décrites. La décision d'angioplastie et de pose d'une endoprothèse repose de fait, le plus souvent, sur des critères de jugement individuel pris en cours d'intervention.

Concernant l'impact du stenting d'une sténose suspectée, il existe peu de données. Deux équipes ont étudié le taux de rethrombose dans les suites d'une prise en charge interventionnelle, selon la mise en place ou non d'une endoprothèse afin de lever un SC suspecté. Les résultats de ces études sont en faveur du stenting avec un taux de rethrombose plus élevé lorsque la compression suspectée est restée non traitée [22, 23].

Il s'agit d'études rétrospectives et il n'existe pas à ce jour de résultat d'études randomisées permettant spécifiquement d'étudier l'impact du stenting, mais surtout de l'absence de stenting d'une sténose suspectée. Dans l'étude randomisée ATTRACT [16], le libre choix du déploiement ou non de stent est laissé aux opérateurs.

\section{Conclusion}

L'absence de sémiologie spécifique et l'hétérogénéité des circonstances diagnostiques font du SC une entité finalement difficile à appréhender. Les progrès considérables de l'imagerie médicale et des techniques interventionnelles semblent permettre une prise en charge efficace et sûre de l'obstruction veineuse, sous réserve d'une évaluation préthérapeutique rigoureuse. Ces résultats encourageants restent toutefois à confirmer par des essais randomisés.

Liens d'intérets: l'auteur déclare ne pas avoir de lien d'intérêt.

\section{Références}

1. May R, Thurner J. Ein Gefassporn in der Vena iliaca communis sinistra als Ursache der uberwiegend linksseitigen Beckenvenenthrombosen [A vascular spur in the vena iliaca communis sinistra as a cause of predominantly left-sided thrombosis of the pelvic veins]. Z Kreislaufforsch $1956 ; 45: 912-22$.

2. Cockett FB, Thomas ML. The iliac compression syndrome. Br J Surg $1965 ; 52: 816-21$.

3. Virchow R. Ueber die Erweiterung kleinerer Gefäfse. Arch Pathol Anat Physiol Klin Med 1851; 3:427-62.

4. Carr S, Chan K, Rosenberg J, et al. Correlation of the diameter of the left common iliac vein with the risk of lower-extremity deep venous thrombosis. J Vasc Interv Radiol 2012; 23 : 1467-72.

5. Oguzkurt L, Ozkan U, Ulusan S, Koc Z, Tercan F. Compression of the left common iliac vein in asymptomatic subjects and patients with left iliofemoral deep vein thrombosis. J Vasc Interv Radiol 2008 ; 19:366-70 (quiz 71).

6. Birn J, Vedantham S. May-Thurner syndrome and other obstructive iliac vein lesions: meaning, myth, and mystery. Vasc Med 2015; 20 : 74-83.

7. Forauer AR, Gemmete JJ, Dasika NL, Cho KJ, Williams DM. Intravascular ultrasound in the diagnosis and treatment of iliac vein compression (May-Thurner) syndrome. J Vasc Interv Radiol 2002; 13 : 523-7.

8. Canales JF, Krajcer Z. Intravascular ultrasound guidance in treating May-Thurner syndrome. Tex Heart Inst J 2010 ; 37 : 496-7.

9. Raju S, Neglen P. High prevalence of nonthrombotic iliac vein lesions in chronic venous disease: a permissive role in pathogenicity. $J$ Vasc Surg 2006 ; 44 : 136-43 (discussion 44).

10. Neglen P, Raju S. Intravascular ultrasound scan evaluation of the obstructed vein. J Vasc Surg 2002; 35 : 694-700.

11. Labropoulos $\mathrm{N}$, Volteas $\mathrm{N}$, Leon $\mathrm{M}$, et al. The role of venous outflow obstruction in patients with chronic venous dysfunction. Arch Surg $1997 ; 132: 46-51$.

12. Neglen $P$, Raju S. Detection of outflow obstruction in chronic venous insufficiency. J Vasc Surg 1993; 17 : 583-9.

13. Binkert CA, Schoch E, Stuckmann G, et al. Treatment of pelvic venous spur (May-Thurner syndrome) with self-expanding metallic endoprostheses. Cardiovasc Intervent Radiol 1998 ; 21 : 22-6.

14. O'Sullivan GJ, Semba CP, Bittner CA, et al. Endovascular management of iliac vein compression (May-Thurner) syndrome. J Vasc Interv Radiol 2000; $11: 823-36$.

15. Karthikesalingam A, Young EL, Hinchliffe RJ, Loftus IM, Thompson MM, Holt PJ. A systematic review of percutaneous mechanical thrombectomy in the treatment of deep venous thrombosis. Eur J Vasc Endovasc Surg $2011 ; 41: 554-65$.

16. Vedantham S, Goldhaber SZ, Kahn SR, et al. Rationale and design of the ATTRACT study: a multicenter randomized trial to evaluate pharmacomechanical catheter-directed thrombolysis for the prevention of postthrombotic syndrome in patients with proximal deep vein thrombosis. Am Heart J 2013; 165 : 523-530 e3.

17. Comerota AJ, Grewal N, Martinez JT, et al. Postthrombotic morbidity correlates with residual thrombus following catheter-directed thrombolysis for iliofemoral deep vein thrombosis. J Vasc Surg 2012; 55 : 768-73. 
18. Seager MJ, Busuttil A, Dharmarajah B, Davies AH. Editor's choice - A systematic review of endovenous stenting in chronic venous disease secondary to iliac vein obstruction. Eur J Vasc Endovasc Surg $2016 ; 51: 100-20$

19. Neglen P, Hollis KC, Olivier J, Raju S. Stenting of the venous outflow in chronic venous disease: long-term stent-related outcome, clinical, and hemodynamic result. J Vasc Surg 2007; 46:979-90.

20. Hartung O, Loundou AD, Barthelemy P, Arnoux D, Boufi M, Alimi YS. Endovascular management of chronic disabling ilio-caval obstructive lesions: long-term results. Eur J Vasc Endovasc Surg 2009; 38 : 118-24.
21. Lou WS, Gu JP, He X, et al. Endovascular treatment for iliac vein compression syndrome: a comparison between the presence and absence of secondary thrombosis. Korean J Radiol 2009; 10 : 135-43.

22. Mickley V, Schwagierek R, Rilinger N, Gorich J, Sunder-Plassmann L. Left iliac venous thrombosis caused by venous spur: treatment with thrombectomy and stent implantation. J Vasc Surg 1998;28: 492-7.

23. Hartung O, Benmiloud F, Barthelemy P, Dubuc M, Boufi M, Alimi YS. Late results of surgical venous thrombectomy with iliocaval stenting. $J$ Vasc Surg 2008; $47: 381-7$.

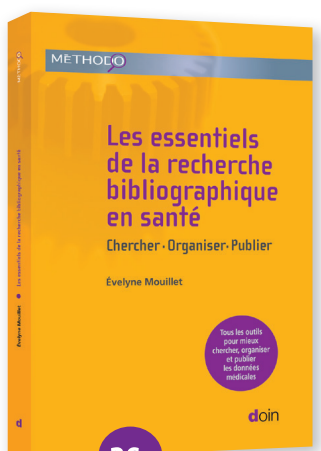

Les essentiels de la recherche bibliographique en santé Chercher • Organiser • Publier

S'adressant à tous les acteurs de santé, cet ouvrage leur apprend à : - conduire une recherche documentaire pertinente,

- sélectionner les documents utiles,

- gérer une veille bibliographique,

- connaître les règles de la rédaction bibliographique.

Evelyne Mouillet

Bibliothécaire, chargée d'enseignement / Institut de santé publique, d'épidémiologie et de développement (ISPED), Université de Bordeaux

Collection Méthodo

- Septembre 2016

- $16 \times 24 \mathrm{~cm} / 208$ pages

- ISBN : 978-2-7040-1471-2

\section{doin | DU]}

\section{METHOLO}

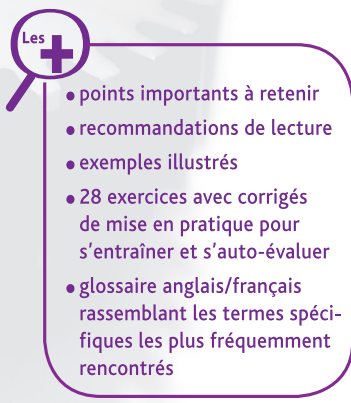

- recommandations de lecture

exemples illustrés

exercices avec corrig

mise en pratique pour

s'entraîner et s'auto-évalue

ques les plus fréquemment

rencontrés

Ouvrage disponible sur www.jle.com 